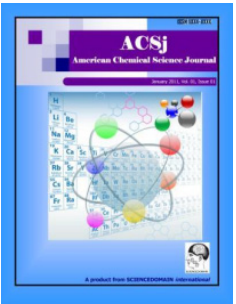

American Chemical Science Journal

1(1): 28-36, 2011

SCIENCEDOMAIN international

www.sciencedomain.org

\title{
Silica Sulphuric Acid Catalyzed Fischer Esterification Reaction under Solventless Condition: A Novel Green Chemical Reaction
}

\author{
A. Rajendran ${ }^{1 *}$ and C. Karthikeyan ${ }^{1}$ \\ ${ }^{1}$ Department of Chemistry, Sir Theagaraya College, Chennai - 21, Tamil Nadu, India.
}

Research Article

Received 11 ${ }^{\text {th }}$ May 2011

Accepted $16^{\text {th }}$ May 2011

Online Ready $21^{\text {st }}$ May 2011

\section{ABSTRACT}

Objectives: The science of chemistry has matured, and green chemistry emphasizes the creative use and design of chemicals. The main objective of this work is to carryout Fisher esterification reactions using silica sulphuric acid ionic liquid in such a way that they have little or no pollution potential or environmental risk and are both economically and technologically feasible. The aim is to prove the catalytic efficiency of silica sulphuric acid ionic liquid as a green chemical.

Study design: Fisher esterification reaction using silica-sulphuric acid ionic liquid as a catalyst under solventless condition.

Place and Duration of Study: Department of Chemistry, Sir Theagaraya College, Chennai-21, Tamilnadu, India, between July 2009 and September 2010.

Methodology: It involves five stages. First stage is the synthesis of silica sulphuric acid ionic liquid in an eco-friendly way by the reaction of silica gel and chlorosulphonic acid .It is interesting to note that the reaction is easy, clean and not requiring any work-up procedure. Second stage is the Fisher esterification reaction of a variety of carboxylic acids and alcohol utilizing silica sulphuric acid. Third stage is the purification of products by chromatographic techniques. The fourth stage id the characterization of all the products by spectral methods. Fifth stage is the comparison of the catalytic efficiency of silica sulphuric acid with the conventional sulphuric acid catalyst.

Results: The silica supported sulphuric acid is bench top reagent which is easy to handle with better accessibility of the reactants to the active sites. Fisher esterification reactions were completed with excellent yields $(97-99 \%)$ within $1.5-8 \mathrm{hr}$ at the temperature range of $80-135^{\circ} \mathrm{C}$. The reaction does not require any additional catalyst because the ionic liquid itself acts as a catalyst and solvent as well in these experiments, the products were isolated and the remaining catalyst was washed and reloaded with fresh substrates. When 
similar reactions were carried out in sulphuric acid (conventional acid catalyst), a mixture of products was obtained in poor yields One of the greatest advantages of the reagent $\mathrm{SiO}_{2} \cdot \mathrm{OSO}_{3} \mathrm{~h}$ is that it is recoverable and reusable for several times without potential loss in its catalytic activity.

Conclusion: The results indicated that the silica sulphuric acid exhibited excellent catalytic activity for the esterification reaction and better yield (with high degree of purity) under mild reaction conditions than those reactions without ionic liquid. After the reaction was over, the ionic liquid was regenerated and reused with no appreciable change in activity. In conclusion the present method is a very efficient and selective protocol for esterification reactions of carboxylic acids with alcohols and a high yield synthesis of the required ester in the presence of reversible and environmentally benign catalyst. Simple work-up procedure, including washing the mixture followed by evaporation of the solvent is another advantage of this method.

Keywords: Green synthesis; ionic liquid; silica sulphuric acid; Fisher esterification reaction; solvent less reaction; Green chemical reaction;

\section{ABBREVIATIONS}

ILS: Ionic Liquids; TSILs: Task Specific Ionic Liquids; RTILs: Room Temperature Ionic Liquids;

\section{INTRODUCTION}

Green chemistry, environmentally benign chemical synthesis, and alternative synthetic pathways for pollution prevention, benign by design: these phrases all essentially describe the same concept (Anastas and Warner, 1998). Green chemistry is the utilization of a set of principles that reduces or eliminates the use or generation of hazardous substances in the design, manufacture, and application of chemical products. Green chemistry involves the design of chemical syntheses, and the use of material reagents and processes that prevent pollution. Green chemistry is not necessarily environmental chemistry, although it may involve some of this. Pollution is usually defined as chemical pollution. Most often the approach utilized in the laws has been one of controlling the amount of release of a chemical or controlling the final concentration of that chemical in the medium to which it was released.

Green chemistry deals with the above situation somewhat differently: preventing the releases (Kumar, 2009). The goals of preventing pollution through use of less hazardous and environmentally benign chemistry are achievable. The issues cannot simply be addressed by minor tinkering with current processes to improve their performance, although that is one of many of the strategies that create new paradigms in chemistry (Trost, 1998).

Rather than controlling the concentrations or releases of a particular chemical substance through engineering controls or waste treatment procedures, green chemistry, instead, changes the intrinsic nature of the substances themselves so as to reduce or eliminate the hazard posed by the substances (Rajendran, 2010). This is particularly appealing from a science philosophy perspective because of the nexus between classical chemistry and mechanistic toxicology. To accomplish this, the green chemist must balance the molecular 
features that are needed for the tasks to be performed with those molecular features that are responsible for the intrinsic hazard of the substance.

Solvents incorporate all the concepts presented thus far. The science of chemistry has matured, and green chemistry emphasizes the creative use and design of chemicals. This necessarily involves the utilization of existing knowledge, wielded in a novel manner by the chemist (Trost, 1998). Solvents are a necessary component. Green chemistry incorporates the design, manufacture, and use of chemicals and chemical processes into its practices in such a way that they have little or no pollution potential or environmental risk and are both economically and technologically feasible. This teleological view of green chemistry is counter to traditional expectations in chemistry. Chemists should not be ignorant nor can they claim to be unaware of the effects of the science in which we are engaged (Anastas and Warner, 1998).

Green chemistry reduces the hazards associated with chemical use. It is the use of chemistry techniques and methodologies that will reduce or eliminate the use or generation of feed stocks, product, byproducts, solvents, reagents, and so on, that are hazardous to human health or the environment (Papasavva and Moomaw, 1997). Green chemistry, by espousing rigorous scientific principles, will afford new opportunities and possibilities in this science, which will offer to the world environmentally benign alternatives to existing hazards found in chemistry. As our chemical knowledge has expanded, the art of chemistry has uncovered more fundamental principles of the relationships between structure and industrial function. This new knowledge must force chemists to incorporate this into how we evaluate the success of any chemical endeavor (Kumar, 2009). As time passes and our awareness of our global environment increases, the world's populace becomes acutely cognizant of the detrimental effects resulting from human endeavors. Incorporated into its assessment of the success of its chemical activities, an "environmental barometer" will demonstrate the commitment of chemistry to the environment. Green chemistry has as its aim to conduct its chemical activities in such a way as to minimize the generation of waste and to continually increase the beneficial environmental effects relative to previous practices. The text herein will provide the first authoritative description of solvents for those interested green chemistry. The definition of solvents will be quite broad, as to include the full range of commercial solvents (e.g., cleaners) through industrial solvents to those used in "academic" settings.

Solvents, explicitly or implicitly, are a critical component of chemistry. Indeed, in the area of chemical reactions, including solventless systems, it is impossible to neglect the role solvents play. This is intended to provide scientists and nonscientists interested in green chemistry a reference to the considerations for solvents in this new and growing area. The concept of designing and using safer solvents brings a new dimension to the chemical enterprise; namely, a far greater consideration of the impact of a new or existing solvent on human health and environment throughout the chemical's life cycle of manufacture, use, and disposal. This involves not only important considerations by chemists regarding the design of new solvents, but, equally important, it includes the reconsideration of and redesign of a wide range of existing chemicals, reactions, and processes.

In recent years, environmentally-friendly reaction processes have vigorously been studied from the stand point of green chemistry. For example, Oxidation reactions with the air, or reaction in water, supercritical fluids, and fluorous solvents are cited (Anastas and Warner, 1998; Rajendran, 2010; Kumar, 2009). Most recently, ionic liquids have gained much attention as green solvents for organic synthesis (Rajendran, 2010). 
Ionic liquids are liquids at or near the room temperature; they can also be considered organic salts. In recent years, applications of ionic liquids (ILs) in organic synthesis have attracted considerable attention due to their special properties such as good solvating capability, wide liquid range, non-inflammability, negligible vapor pressure, easy of recycling, high thermal stability and rate enhancers. Also, ILs as environmentally benign media for catalytic processes and much attention has currently been focused on organic reaction catalyzed by ILs have been reported with high performance. Ionic liquids have been shown to have applications in the field of catalysis, metal deposition, extraction and batteries. Their cost and sensitivity to oxygen and moisture have however, limited their applications to these areas. We have developed a range of ionic liquids that are inert to air and water and are similar in cost to common organic solvents (Rajendran, 2010). These fluids have negligible vapor pressures, large liquid ranges and are environmentally benign. We are currently studying the physical and chemical characteristics of these liquids together with preliminary investigations to assess their suitability for a number of chemical and electrochemical applications. The recent interest surrounding ionic liquids in regards to green chemistry has largely been a result of the fact that they have no measurable vapor pressure and hence emit no volatile organic compounds. But replacing volatile organic solvents is just part of the story indeed, ionic liquids are very popular materials and they enjoy a plethora of applications in various domains of physical sciences.

Research has been carried out on a number of organic reactions in ionic liquids e.g. FriedelCraft acylations, Diels-Alder reactions, aldol reactions, Fischer - indole synthesis (see below), in these cases the ionic liquid acts as both the solvent and as a catalyst. Researchers have studied several examples of the Fischer indole synthesis in the ionic liquid choline chloride: $2 \mathrm{ZnCl}_{2}$ and find that after heating to $90^{\circ} \mathrm{C}$ for two hours the yields are uniformly good. These conditions are milder than the typical cyclization which involves heating in polyphosphoric acid at $100^{\circ} \mathrm{C}$. Thus they have established that they can reduce the amount of acid from use of mineral acid as a solvent to use of a Lewis acid in just over stoichiometric amounts (1.5 equivalents currently) using milder conditions giving yields that are at least as good or better than the conventional process. The work-up procedure just involves adding water which dissolves the ionic liquid which is removed by filtration. Hence, no organic solvent is necessary for the reaction or work-up (Trost, 1998).

In several industrially important processes (e.g., nitration, condensation, esterification, etc.) a large excess of sulphuric acid is required because the water by-product slows the reaction down by diluting the acid. At the end of these processes, a large amount of "spent acid" is obtained which, in batch reactions, is usually neutralized and disposed of, which in continuous processes, it has to be recycled by complex techniques. Also, the separation of the products from the acid is often a difficult and energy consuming process that habitually implies a basic aqueous work-up. Moreover, sulphuric acid is corrosive and dangerous to transport and handle. Consequently and reduction in the amount of sulphuric acid needed dangerous and/or any simplifications in handling procedures would be highly convenient in terms of risk reduction, economic advantages and environment protection (Zhang et al., 2005). On the other hand there is intense current research and general interest in heterogeneous system because of its perceived opportunities. Such systems present for basic research and because of its unquestioned importance, such systems have remarkable applications in industry and in developing technologies (Wang et al., 2006; Shelton et al., 2009). In continuation of our studies on the application of inorganic acidic salts, we found that silica gel reacts with sulphuric acid to give silica-sulphuric acid. It is interesting to note that the reaction is easy and clean and not requiring any work-up procedure (Scheme 1). 
Esterification reaction is a powerful for the formation of many synthetically important esters which acts as the precursors in synthesis several others industrially, medically important compounds (Smith and March, 2003). Esterification is an equilibrium reaction which can be driven in the forward direction by using one of the reactants- acid or alcohol in excess or by removing the product water from the reaction by distillation or by use of a dehydrating agent or by molecular sieve (Larock, 1999). A common procedure is to carryout the esterification in the presence of benzene and a catalytic quantity of a mineral acid like $\mathrm{H}_{2} \mathrm{SO}_{4}$. A Deen-stark apparatus was used for distilling out water as an azoetrope. These traditional acid or base catalyzed esterification reactions suffer from reverse reaction.) In continuation of our studies on the application of silica sulfuric acid as a versatile and stable solid acid in organic synthesis, we wish to report an efficient and selective method for esterification reactions of some aromatic acids aliphatic acids with ethanol and methanol under solvent free conditions (Ganeshpure et al., 2007; Shelke et al., 2009; Sahoo et al., 2006).

\section{EXPERIMENTAL DETAILS}

\subsection{Materials and Reagents}

All chemicals (AR grade) were commercially available and used without further purification unless otherwise stated. The uncorrected melting points of compounds were taken in an open capillary in a paraffin bath. UV-VIS spectra were recorded in double beam Uv-vis spectrophotometer (Lambda series model, Shimadzu make), IR spectra were recorded on Perkin-Elmer FT-IR spectrophotometer in $\mathrm{KBr}$ disc. ${ }^{1} \mathrm{H}-\mathrm{NMR}$ and ${ }^{13} \mathrm{C}-\mathrm{NMR}$ spectra were recorded on a high resolution $400 \mathrm{MHz}$ NMR spectrophotometer (Bruckner model) in $\mathrm{CDCl}_{3} /$ DMSO- $\mathrm{D}_{6}$ as a solvent and chemical shift values are recorded in $\delta$ units (ppm) relative to TMS as an internal standard.

\subsection{Preparation of Silica Sulphuric Acid Ionic Liquid}

The ionic liquid $\mathrm{SiO}_{2} \cdot \mathrm{OSO}_{3} \mathrm{H}$ was prepared according to the reported procedure in an ecofriendly way by the reaction of silica gel and chlorosulphonic acid (Ganeshpure et al., 2007; Sahoo et al., 2006). It is interesting to note that the reaction is easy, clean and not requiring any work-up procedure. A $500 \mathrm{ml}$ suction flask was used. It was equipped with a constantpressure dropping funnel containing chlorosulphonic acid $(23.3 \mathrm{~g}, 0.2 \mathrm{~mol})$ and a gas inlet tube for conducting $\mathrm{HCl}$ gas over an absorbing solution (water). It was charged with silica gel $(60.0 \mathrm{~g})$ and chlorosulphonic acid was added drop wise over a period of 30 minutes at room temperature. After the addition was completed the mixture was shaken well for 30 minutes, a white solid of silica sulphuric acid $(76.0 \mathrm{~g})$ was obtained (Scheme 1).

$$
\mathrm{SiO}_{2}-\mathrm{OH}+\mathrm{ClSO}_{3} \mathrm{H} \underset{\text { Temperature }}{\stackrel{\text { Room }}{\longrightarrow}} \mathrm{SiO}_{2}-\mathrm{OSO}_{3} \mathrm{H}+\mathrm{HCl} \uparrow
$$

Scheme 1. Synthesis of ionic liquid [silica-sulphuric acid]

\subsection{General Procedure for Fisher Esterification Reaction with Silica Sulphuric Acid}

In typical esterification reactions carboxylic acid $(0.01 \mathrm{~mol})$, ethanol $(0.01 \mathrm{~mol})$ and ionic liquid $\left(\mathrm{SiO}_{2}-\mathrm{OSO}_{3} \mathrm{H}\right)(0.005 \mathrm{~mol})$ were taken in a round-bottom flask fitted with a reflux condenser. The contents were heated on a boiling water-bath for different length of times. 
The RB flask is then fitted with Young's still head ( 4 bulbs), and the contents fractionally distilled using water condenser first (unreacted ethanol distills over). When the required temperature was reached for different reactions, the receiver was changed to a china dish and the distillation was continued using air-condenser. The distillate was got solidified in the china dish. It was then dried and the yield was noted. Products were characterized by comparison of other spectroscopic data (UV-VIS, FT-IR, ${ }^{1} \mathrm{H}-\mathrm{NMR}$ and ${ }^{13} \mathrm{C}-\mathrm{NMR}$ ) and physical properties with these reported in the literature (Smith and March, 2001). The products were identified using ${ }^{1} \mathrm{H}-\mathrm{NMR}$ in $\mathrm{CDCl}_{3}$ with TMS as reference $(300 \mathrm{MHz})$ and by FT-IR on a Shimadzu (Model 8201 pc) spectrophotometer using $\mathrm{KBr}$ pellets in a frequency range and by a double beam UV-Vis. Spectrophotometer [Elico make Model SL-191]. The ionic liquid in the filtrate was separated from unreacted starting materials by decantation or by filtration. Then the ionic liquid was dried at $100^{\circ} \mathrm{C}$ for $5 \mathrm{hrs}$. to remove water and the silica-sulphuric acid was reused.

\section{RESULTS AND DISCUSSION}

Silica-sulphuric acid has been used in small and long scale reactions as an acid catalyst. In many synthetic reports $\mathrm{SiO}_{2} \cdot \mathrm{OSO}_{3} \mathrm{H}$ has been used. The silica supported sulphuric acid is bench top reagent which is easy to handle with better accessibility of the reactants to the active sites. Exposure of high surface area oxides such as $\mathrm{Si}-\mathrm{O}-\mathrm{Si}$ to $\mathrm{H}_{2} \mathrm{SO}_{4}$ at room temperature results in irreversible adsorption. The resulting surface possess surface species such as $\mathrm{Si}-\mathrm{O}_{2}-\mathrm{SO}_{3}-\mathrm{H}^{+}$. The esterification of several carboxylic acids (both aliphatic and aromatic carboxylic acids) with ethanol was carried out using $\mathrm{SiO}_{2} \cdot \mathrm{OSO}_{3} \mathrm{H}$ was a catalyst.

Excellent yields were noted because of the good solubility of the acids and ethanol in silicasulphuric acid while the esters are almost immiscible with ionic liquid. This novel ionic liquid $\mathrm{SiO}_{2} . \mathrm{OSO}_{3} \mathrm{H}$ which was screened as a catalyst for esterification of carboxylic acids and ethanol could be easily reused after removal of water under vacuum. Furthermore, the present purification procedure could be used for the clean-up of a contaminated ionic liquid by extracting it into a polar extractant to form an extract containing the ionic liquid, water traces were removed from the extract. Then, the extract was filtered and the high-purity ionic liquid was recovered from the filtered extract. The catalytic activity of this extracted $\mathrm{SiO}_{2} . \mathrm{OSO}_{3} \mathrm{H}$ was investigated. Its catalytic activity was found to be still very high even after half-a-dozen times, demonstrating that $\mathrm{SiO}_{2} \cdot \mathrm{OSO}_{3} \mathrm{H}$ can be reused in esterification reactions, without any environmental discharges.

The esterification results of several different acids with ethanol were presented in Table 1. It could be found that the ionic liquid $\mathrm{SiO} 2 . \mathrm{OSO} 3 \mathrm{H}$ exhibited its remarkable activity for esterification of aromatic acids gave lower yields compared with those of aliphatic acids. No by-product was noticed. When similar reactions were conducted in sulphuric acid alone, the products were obtained in poor yields. The promoting effect of silica sulphuric acid in these reactions was shown by performing some reactions on the absence of the reagent (Table 1). Table 1 depicts the efficiency of silica sulphuric acid. In this experiment the product was isolated and the remaining catalyst was washed and reloaded with fresh substrates for further runs. 
Table 1. Results of esterification of different acids and ethanol in $\mathrm{SiO}_{2} \cdot \mathrm{OSO}_{3} \mathrm{H}$ (with ionic liquid)

\begin{tabular}{llccc}
\hline Entry & Carboxylic Acid & Time (hr.) & $\mathbf{T}^{\mathbf{0}}(\mathbf{C})$ & Yield (\%) $^{\mathrm{a}}$ \\
\hline 1 & Acetic acid & 1.5 & 110 & 98 \\
& & $3.0^{\mathrm{b}}$ & $126^{\mathrm{b}}$ & $89^{\mathrm{b}}$ \\
2 & Benzoic acid & 6.0 & 80 & 97 \\
& & $9.0^{\mathrm{b}}$ & $90^{\mathrm{b}}$ & $88^{\mathrm{b}}$ \\
3 & Monochloroacetic acid & 2.0 & 120 & 97 \\
& & $3.0^{\mathrm{b}}$ & $130^{\mathrm{b}}$ & $87^{\mathrm{b}}$ \\
4 & \multirow{2}{*}{ Salicylic acid } & 8.0 & 135 & 99 \\
& & $10^{\mathrm{b}}$ & $150^{\mathrm{b}}$ & $90^{\mathrm{b}}$ \\
& & 8.0 & 130 & 98 \\
& Cinnamic acid & $10^{\mathrm{b}}$ & $140^{\mathrm{b}}$ & $91^{\mathrm{b}}$ \\
\hline
\end{tabular}

a Isolated yield based upon the starting carboxylic acid;

${ }^{b}$ Effect of sulphuric acid (conventional catalyst) on Fisher esterification reaction;

Table 2. Reuse of $\mathrm{SiO}_{2} \cdot \mathrm{OSO}_{3} \mathrm{H}$ in the synthesis of Methyl benzoate

\begin{tabular}{cccc}
\hline Run & Time $(\mathbf{h})$ & $\mathbf{T}^{\mathbf{0}} \mathbf{( C )}$ & Yield (\%) $^{\mathbf{a}}$ \\
\hline 1 & 6.0 & 80 & 90 \\
2 & 6.0 & 80 & 90 \\
3 & 6.0 & 80 & 89 \\
4 & 6.0 & 80 & 90 \\
5 & 6.0 & 80 & 89 \\
6 & 6.0 & 80 & 89 \\
\hline \multicolumn{4}{r}{ alsolated yield based upon the starting carboxylic acid } \\
\end{tabular}

When the results presented in Table 1 are compared in terms of Time, Temperature and yield, it is quite obvious that esterification reactions carried out in silica-sulphuric acid showed better yield with higher purity in lesser time and lesser temperature than the conventional catalyst, sulphuric acid. This is because of the fact that silica-sulphuric acid serves as a much better proton source than sulphuric acid itself. The $\mathrm{SiO}_{2} \cdot \mathrm{OSO}_{3} \mathrm{H}$ is miscible with water and immiscible with ethers, so the esterification reactions proceeded smoothly for completion even without simultaneous removal of the produced water, even though esterification is a reversible reaction.

After the completion of the esterification reactions, Silica-Sulphuric acid was recycled and regenerated by removal of water in vacuum. It was reused repeatedly over a half dozen times in esterification reaction of benzoic acid with ethanol. The results are presented in table 2 , which suggest that the catalytic activity of $\mathrm{SiO}_{2} \cdot \mathrm{SO}_{3} \mathrm{H}$ was not remarkably changed.

\section{CONCLUSION}

The esterification reactions of different acids (both aliphatic and aromatic acids) and ethanol were investigated for their efficiency with $\mathrm{SiO}_{2} \cdot \mathrm{SO}_{3} \mathrm{H}$ as ionic liquid and without ionic liquid 
(with simply $\mathrm{H}_{2} \mathrm{SO}_{4}$ ). The results indicated that the silica sulphuric acid exhibited excellent catalytic activity for the esterification reaction and better yield (with high degree of purity) under mild reaction conditions than those reactions without ionic liquid. After the reaction was over, the ionic liquid was regenerated and reused with no appreciable change in activity. In conclusion the present method is a very efficient and selective protocol for esterification reactions of carboxylic acids with alcohols and a high yield synthesis of the required ester in the presence of reversible and environmentally benign catalyst. Simple work-up procedure, including washing the mixture followed by evaporation of the solvent is another advantage of this method.

\section{ACKNOWLEDGMENTS}

The authors immensely thank the UGC, New Delhi for the financial support rendered for the major research project [F. No. 35-147 / 2009 (SR)] .The authors also thank the principal and the management of Sir Theagaraya College, Chennai-21, Tamil Nadu for their constant encouragement and support given.

\section{REFERENCES}

Ahluwalia, V.K., Aggarwal, R. (2006). Organic Synthesis - Special Techniques, 2nd Eds. Narasa Publishing house, pp. 103-139.

Anastas, P.T., Farris, C.A., Eds. (1994). Benign by Design: Alternative Synthetic Design for Pollution Prevention, American Chemical Society, Washington, DC.

Anastas, P.T., Warner, J.C. (1998). Green Chemistry: Theory and Practice, Oxford University Press, Oxford, UK.

Dailei, Song, Yongli, Chen, Runxia, Wang, Chunsheng, Liu, Heng, Jiang, Genxiang, Luo. (2009). Crossed - Aldol condensation of cycloalkanones with aromatic aldehydes catalyzed by copper(II) trifluoracetate. Preparative Biochemistry and Biotechnology, 39(2), $201-207$.

Ganeshpure, P.A., George, G., Das, J. (2007). Application of triethylammonium salts as ionic liquid catalyst and medium for Fisher esterification, ARKIVOC, 8, 273-278.

Kumar, V. (2009). An Introduction to Green Chemistry, Vishal Publishing Co, India, pp. 13

Larock, R.C. (1999). Comprehensive Organic Transformations, VCH: New York, pp. 576.

Papasavva, S., Moomaw, W.R. (1997). International Environmental Affairs, 9, 219-231.

Rajendran, A. (2010). Glimpses of Green Chemistry: Prevention of Pollution by Chemical Design, S. Viswanathan printers and publishers Pvt. Ltd., India (In Press).

Sahoo, S., Joseph, T., Halligudi, S.B. (2006). Mannich reaction in Bronsted acidic ionic liquid: a facile synthesis of amino carbonyl compounds, J. Mole. Catal. A: Chemical, 244, 179-182.

Shelke, K.F., Sapkal, S.B., Madje, B.R., Shingate, B.B., Shingare, M.S. (2009). Ionic liquid promoted an efficient synthesis of 5 - arylidene - 2,4- thiazolidinedione. Bull. Catal. Soc. India, 8, 30-34.

Shelton, R.A., Arends, I., Hanefeld, U. (2009). Green Chemistry and Catalysis, Wiley-VCH, New York, N.Y.

Smith, M.B., March, J. (2001). Advanced Organic Chemistry, Reactions, Mechanisms and Structure; John Wiley \& Sons: New York, pp. 1218- 1224.

Trost, B. M. (1991). Sci., 254, 1471-7. 
Wang, C., Guo, L., Li, H., Wang, Y., Weng, J., Wu, L. (2006). Preparation of simple ammonium ionic liquids and their application in the cracking of dialkoxypropanes. Green Chem., 8, 603-607.

Zhang, G.L.E., Chen, Z.C., Hu, Y., Zheng, Q.G. (2005). Organic reactions in Ionic Liquids: Ionic liquid - promoted three component Condensation of Benzotriazole with Aldehyde and Alcohol. Chin. Chem. Lett., 16(2), 155-158.

(c) 2011 Rajendran \& Karthikeyan; This is an Open Access article distributed under the terms of the Creative Commons Attribution License (http://creativecommons.org/licenses/by/3.0), which permits unrestricted use, distribution, and reproduction in any medium, provided the original work is properly cited. 